\title{
Network Utility Maximization for Information Frequency Adaptation in Vehicular Networks
}

\author{
Hayder M Amer ${ }^{1}$, Hawraa H. Al Mohammed ${ }^{2}$, Lyudmila Mihaylova ${ }^{3}$, Marcos Rodrigues ${ }^{4}$ \\ \{hayder.amer@stu.edu.iq ${ }^{1}$, hawraahm89@gmail.com², 1.s.mihaylova@sheffield.ac.uk ${ }^{3}$ \} \\ Computer System Department, Technical Institute of Basra, Southern Technical University-Iraq ${ }^{1}$ \\ Sheffield Hallam University, Computer and Networking department, Sheffield, United Kingdom ${ }^{2}$ \\ Department of Automatic Control and Systems Engineering, University of Sheffield, Sheffield, \\ United Kingdom ${ }^{3}$
}

\begin{abstract}
The increment in traffic jams in vehicular environments has a vital influence on the wireless channel. This is due to broadcasting many application messages among communicated vehicles. This has generated a data wireless channel congestion problem. This problem influences the efficiency and performance of the network due to the large data collision, high messages loss, and low throughput which in turn decreases the awareness and safety in the vehicular environments. In this paper, a new efficient broadcast approach has been developed based on the network utility maximization method to adjust vehicle broadcasting rate and mitigate overhead on the wireless channel. The results from the simulation scenario have shown that the developed broadcast protocol enhances vehicular network performance by reducing channel congestion and improves network attributes such as delay, throughput, messages loss, and total channel occupation time as compared to other tested approaches.
\end{abstract}

Keywords: Vehicular networks, IoV networks, Wireless channel congestion, Optimization approach.

\section{Introduction}

Intelligent Transportation Systems (ITSs) are considered a powerful system for enhancing vehicles mobility in smart cities [1]. Providing vehicles with communication devices has introduced a new concept for an innovative approach for the components of safety applications in the vehicular communication environment, path planning, and entertainment. This can happen with the incorporation of multiple technologies and devices installed inside the vehicle [2]. The ITSs consolidated a broad range of control schemes such as wireless communication tools and technologies that can be utilized to generate data and announcement of traffic messages to boost road infrastructure and vehicles mobility which in turn helps to improve awareness and safety drivers.

The Internet of Things (IoT) [3] has been regarded as an attractive challenge for the transportation study society and it presents a novel area for ITSs. IoT includes many connected devices and Vehicular Ad-hoc Networks (VANETs) are considered as one of the major elements. VANETs included different wireless communication systems such as Vehicle to Vehicle (V2V) and Roadside Units (RSU) or Vehicle to Infrastructure (V2I) communication systems [4]. These systems are wireless sensors installed inside vehicles or deployed on the 
road segments to collect and broadcast traffic-related information. VANETs can be employed to observe the conditions of the vehicular environment such as accidents and vehicles flow on the road segments. The protocol that is used to govern and support the communication among connected vehicles is called the Wireless Access of Vehicular Environment (WAVE) protocol [5]. However, increasing the number of communicated messages among drivers leads to the problem of data channel congestion in VANETs. This occurs due to the absence of centralized and stable topology and sending many messages at a fixed rate through the communication channel. This also increments the amount of battled vehicles to obtain the communication channel which influences the time that is required to send the safety messages and leads to a decrease the awareness in the vehicular environments. The problem of channel congestion hurts the parameters and performance of the network [6].

Many protocols and approaches have been developed to improve vehicular network performance in the presence of roads congestion and dynamic scenarios. Periodic messages adaptation strategies have been developed in [7] and [8]. The authors in [7] have introduced a beacon adaptation strategy that utilizes the road lanes to optimize vehicles transmission rate. The more multiple lanes on the road segment, the less the sending rate of the nodes. However, this approach has not calculated the real number of vehicles on the road sections.

In [8], a decentralized beacon modification strategy has been proposed that employs the investigation of the effects of multiple performance measurements such as orientation, quantity, and vehicles transportation conditions on the adaptation of the beacon rate. Nevertheless, the implied approach examines the effects of these parameters separately on the channel congestion rather than joining these measurements to obtain the adapted transmission rates. Moreover, the improper drop of periodic information affects the traffic data that must be propagated among drivers.

The authors in [9] have proposed an adaptation model based on a theoretical analysis to indicate the performance of IEEE 802.11p WAVE protocol and a retransmission strategy of the traffic data is introduced to promote the reliability of VAENTs. Nevertheless, the retransmission is initiated once a failed delivery has occurred. This approach causes data redundancy and overloads the wireless communication channel.

In [10], an approach that is called Utility-Based Packet Forwarding and Congestion Control (UBPFCC) has been introduced to enhance the sending frequency of beacon messages. This approach uses the size and cost function of data to adapt the rate of beacon packets. The UBPFCC employs a cost function specified for every vehicle to dynamically determine the rest of sending frequency to the vehicles. This approach gives the vehicle with high-cost function a high sending rate while those with the lower values broadcast data at low rates. However, this strategy has not estimated the density of vehicles on the road sections when calculating the cost function which has significant effects on the broadcast rate of the traffic information.

A data rate adaptation approach that uses game theory has been presented in [11]. A noncooperative game approach has been utilized to module the problem of channel congestion and the existence of the optimal solution has been proved mathematically. The optimal data rate solution has been estimated via the KKT-Lagrange multiplier method. However, this approach has not considered the mobility of vehicles in the formulated cost function which makes it valid for only dense scenarios while the awareness of vehicles has been affected in the sparse environment.

Hence, this paper develops a novel efficient broadcast rate strategy for VANETs by applying Network Utility Maximization (NUM) method. The developed strategy implements a broadcast protocol to regulate the problem of wireless congestion in the vehicular network by using traffic control to enhance adequately the performance and measurement parameters of the network. 
The suggested strategy essential participation:

1. A novel strategy of information rate adaptation that is called NUM-Date Rate Control (NUM-DRC) has been developed. This strategy adapts the sending rate based on the optimization method to alleviate wireless channel congestion. The sending rate and channel occupancy time have been used in the cost function to obtain solicited balance.

2. 2. The presence of a single optimal solution has been proved in this work for the vehicular network.

3. Priorities for the transmitted applications have been considered in this work to differentiate between high priority messages and low priority messages.

4. Lagrange multipliers have been applied to resolve the optimization dilemma and KTT conditions are used to find the optimal solution (information optimal sending rate).

5. A comprehensive performance evaluation is carried out for the proposed approach. This involves measuring the proposed strategy over a highway scenario. The proposed strategy is compared with other implemented strategies from the literature. The obtained outcomes confirm that the suggested strategy is adjusted to efficiently optimize the rates of traffic information to mitigate the data congestion problem in a vehicular network.

The remainder of the paper is structured as follows: In Section 2 gives details of the optimization method. Section 3 gives the data rate optimal solution calculation. The section shows 4 the simulation scenario and the evaluation of the proposed approach. Finally, Section 5 concludes the paper.

\section{Information Rate Optimization Approach}

The NUM optimization method has been applied in this paper to propose a novel adaptation approach of the disseminated information rate that is called NUM-DRC. This approach applies the theory of optimization problem to form the problem of information rate adaptation and the target is to maximize the cost function which leads to finding the optimal solution for each vehicle in the network. Here, let $V=\left\{v_{j}, \mathrm{j}=1,2, \cdots, \mathrm{m}\right\}$ represent a group of communicated vehicles that are driving on the road segment and contended with each other to access the wireless channel to broadcast safety messages at a maximum rate in selfish behaviour. Therefore, this paper assumes that:

1. Each vehicle in the network has a sized buffer of $B$ messages

2. Each communicated vehicle in the network has two kinds of safety application messages $A_{p}=\left\{a p_{n} ; n=1,2\right\}$ that are: (i) Periodic messages or beacon is sent periodically and include the information that is related to the traffic status, (ii) Emergency messages are transmitted once an accident occurs or emergencies appear and they disappear when the event fades.

According to the queuing theory [12], [13], the channel congestion and buffer overload happen when vehicles' broadcasting rate is higher than the channel capacity or many vehicles send data at the same time without considering other vehicles' buffer size or channel capacity limitation. Here, the problem is to send data at a rate in a significant approach that guarantees fairness 
among nodes and reduces channel congestion without decreasing awareness among the drivers. This paper utilizes two factors in the formulated utility $U F_{j}\left(R_{j}^{a p_{n}}\right)$ that are:

1. Messages rate $R_{j}^{a p_{n}}$ : It depicts the broadcasting rate for each vehicle in the network. The utility functions have been formed by several representations for example exponential, logarithmic, and linear [14]. This paper utilizes the exponential function to represent the message rate function of vehicle profit. This is because the exponential function has a rigorous concave shape that makes it a distinctive property. In addition, the second derivative of this function is negative. Thus, the vehicle $v_{j}$ message rate function is expressed as follows:

$$
M S_{j}\left(R_{j}^{a p_{n}}\right)=\left(1-e^{\left(1-R_{j}^{a p_{n}}\right)}\right)
$$

2. Price function: In the communication network, the pricing or penalty cost is considered as an effective approach to enhance the network resources utilization [15]. The suitable pricing strategy controls the redundant use of the network resources among contending nodes when the data channel congestion occurs in the network. Here, the Channel Occupancy Percentage (COP) has been utilized as a price that demands to be paid by every vehicle when the congestion happens. The COP has been estimated according to [16] as follows:

$$
C O P_{j}=\sum_{j=1}^{m} h_{k j} R_{j}^{a p_{n}}
$$

where $h_{k j}$ function depicts the likelihood that a definite transmission generates a received signal strength higher than threshold power transmission of the carrier sense at a specific location from the transmitter and it is given by:

$h_{k j}=\frac{r\left(m, \frac{m C S_{T}}{\Omega}\right)}{r(m)} \times T_{\text {frame }}$

Where $T_{\text {frame }}$ is the time needed to transmit a safety application packet, $\Upsilon$ is the gamma function, $m$ is the Nakagami fading parameter, $C S_{T}$ represents the threshold power transmission of the carrier sense and $\Omega$ represents the average received signal strength at a certain position which can be estimated as follows:

$\Omega=P_{t} \frac{\varphi}{d_{k j}^{\eta}}$

Where $P_{t}$ is the power transmission of the sender, $d_{k j}^{\eta}$ is the gap between the sender and the recipient node, the path loss factor is represented by $\eta$, and $\varphi$ is given by:

$\varphi=\frac{G_{S} G_{r} \lambda^{2}}{(4 \pi)^{2}}$

where $G_{s}$ and $G_{r}$ are the antenna gains of sender and receiver nodes, receptively. $\lambda$ is the wavelength. 
Therefore, each player will pay a cost based on its $C O P$ function, if the $C O P$ has a high value this affects the vehicle awareness due to increasing the congestion on the wireless channel. On the other hand, a lower $C O P$ value means a decrease in channel congestion and increases the opportunity to transmit traffic data at a high frequency.

The utility of the price function $P R_{f}$ is given by:

$$
P R_{f}\left(R_{j}^{a p_{n}}\right)=C O P_{j}
$$

Each vehicle $v_{j}$ has a cost function represented by $C F_{j}\left(R_{j}^{a p_{n}}\right)$ as follows:

$$
C F_{j}\left(R_{j}^{a p_{n}}\right)=\alpha_{j} M S_{j}\left(R_{j}^{a p_{n}}\right)-\mu_{j} P R_{f}\left(R_{j}^{a p_{n}}\right)
$$

where $\alpha_{j}$ and $\mu_{j}$ are weight factors for vehicle $v_{j}$ the cost function such that $\alpha_{j}$ and $\mu_{j}>0$ for all $j=1,2, \cdots, m$. This paper has used the NUM model to formulate the specified problem as follows:

$$
\begin{gathered}
\max _{R_{j}^{a p_{n}}} \sum_{j=1}^{m} C F_{j}\left(R_{j}^{a p_{n}}\right), \\
\text { subjet to } \sum_{j=1}^{m} R_{j}^{a p_{n}} \leq L, \\
1 \leq R_{j}^{a p_{n}} \leq R_{\max }^{a p_{n}} \quad \forall j=1,2, \cdots, m .
\end{gathered}
$$

where $R_{j}^{a p_{n}}$ is a vector consisting of $R_{1}^{a p_{n}}, \cdots, R_{j}^{a p_{n}}, \cdots, R_{m}^{a p_{n}}, L$ represents the maximum data load and $R_{\max }^{a p_{n}}$ depicts the maximum broadcast rate of a single-vehicle.

\section{Optimal Data Rate Calculation}

The formulated utility function $C F_{j}\left(R_{j}^{a p_{n}}\right)$ has a robustly concave, property and it is unceasingly differentiable function over $R_{j}^{a p_{n}} \geq 0$ for all $j=1,2, \cdots, m$. The formed optimization dilemma in equation (8) has a sole optimal solution according to the optimization theory [17]. Hence, two solutions can be utilized to find the optimal solution in the modeled optimization problem in (8) that are localized or distributed approaches. The localized approaches are efficient in practice because they can obtain the solution quickly [18]. However, this solution has a disadvantage that is an extra communication of cost messages among drivers which in turn drives to overload the channel of the wireless communication. The distributed strategies are more efficient due to the partitioning of the framework into segments that are locally solved to reduce the additional exchange of data among drivers in the network [19]. However, using these methods increase the CPU time that is required to obtain the optimal solution which requires to be rapid in VANETs. This work utilizes the beacon messages to send the congestion information [15]:

$$
\begin{gathered}
\psi_{j}, \zeta_{j} \geq 0, \\
\text { L- } \sum_{j=1}^{m} R_{j}^{a p_{n}} \geq 0,
\end{gathered}
$$




$$
\nabla \sum_{j=1}^{m} C F_{j}\left(R_{j}^{a p_{n}}\right)+\Psi_{j} \nabla\left(R_{\text {out }}^{a p_{n}}-\sum_{j=1}^{m} R_{j}^{a p_{n}}\right)=0
$$

$$
\Psi_{j}\left(\mathrm{~L}-\sum_{j=1}^{m} R_{j}^{a p_{n}}\right), \zeta_{j}\left(R_{m a x}^{a p_{n}}-R_{j}^{a p_{n}}\right)=0
$$

To solve the equation in (9), three unknown's variables $\left(R_{j}^{a p_{n}}, \psi_{j}, \zeta_{j}\right)$ need to be found. Therefore, three states are estimated based on complementarity constraints optimization: Case $1: \psi_{j}=0, \zeta_{j}=0$ and $\left(1 \leq R_{j}^{a p_{n}} \leq R_{\max }^{a p_{n}}\right)$

$$
\begin{gathered}
\alpha_{j}\left(0-(-1) e^{\left(1-R_{j}^{a p_{n}}\right)}\right)-\mu_{j} h_{k j}=0, \\
R_{j}^{a p_{n}}=\ln \left(\frac{\alpha_{j}}{\mu_{j} h_{k j}}\right)-1 \\
\text { Case } 2: R_{j}^{a p_{n}}=1 \text { and } \zeta_{j}=0 \\
\alpha_{j}\left(0-(-1) e^{(0)}\right)-\mu_{j} h_{k j}-\psi_{j}=0, \\
\psi_{j}=\alpha_{j}-\mu_{j} h_{k j}
\end{gathered}
$$

The solution $R_{j}^{a p_{n}}=1$ is considered if the condition $\left(\psi_{j}>0\right)$ satisfied and it is estimated as follows:

$$
\alpha_{j} \geq \mu_{j} h_{k j} \quad \cdots \text { condition } 1
$$

Case3: $R_{j}^{a p_{n}}=R_{\max }^{a p_{n}}$ and $\psi_{j}=0$

$$
\begin{gathered}
\alpha_{j}\left(0-(-1) e^{\left(1-R_{\max }^{a p_{n}}\right)}\right)-\mu_{j} h_{k j}-\zeta_{j}=0, \\
\zeta_{j}=\alpha_{j}\left(e^{\left(1-R_{\max }^{a p_{n}}\right)}\right)-\mu_{j} h_{k j} .
\end{gathered}
$$

The solution $R_{j}^{a p_{n}}=R_{\max }^{a p_{n}}$ is founded, if the condition $\left(\zeta_{j}>0\right)$ satisfied and it is as follows:

$$
\mu_{j} h_{k j} \leq \alpha_{j}\left(e^{\left(1-R_{\max }^{a p_{n}}\right)}\right) \quad \cdots \text { condition } 2
$$

Hence, the global optimum of information sending rate $\left(R_{j}^{a p_{n}^{*}}\right)$ for player $v_{j} ; \forall j \in V$.

$$
R_{j}^{a p_{n}^{*}}= \begin{cases}1-\ln \left(\frac{\mu_{j} h_{k j}}{\alpha_{j}}\right) \text { otherwise } \\ 1 \quad \text { if condition } 1 \\ R_{\max }^{a p_{n}} \quad \text { if condition } 2\end{cases}
$$

\section{Vehicles Broadcasting Rate Allocation Among Safety Application}

VANETs applications require that each vehicle needs to be aware of the priorities of the transmitted messages. Here, this paper assumes that a vehicle, $v_{j}$ hosts two applications 
(beacon and warning messages) with different priorities where $A P=\left\{a p_{n} ; n=1,2\right\}$.Here, $a p_{n}$ denotes the priority of transmitted beacon or emergency messages by vehicles. Therefore, a vehicle with a low value of $a p_{n}$ has a higher priority to transmit traffic information at a high frequency. Once each vehicle determines its transmitted rate $\left(R_{j}^{a p_{n}}\right)$ by using the NUM model. Then, this value is disseminated among safety applications based on their priorities as follows:

$$
\begin{aligned}
& R_{j}^{a p_{n}}=w_{j} R_{j}^{a p_{n}^{*}} \\
& w_{j}= \begin{cases}\frac{\sum_{n=1}^{2} a p_{n}}{(n-1) \sum_{i=1}^{2} a p_{n}} & \text { if } n=2 \\
1 & \text { if } n=1\end{cases} \\
& \sum_{n=1}^{2} w_{j}=1
\end{aligned}
$$

Where $R_{j}^{a p_{n}}$ represents the transmitted rate of safety application $j$ hosted in the vehicle $v_{j}, w_{j}$ is preference weight parameter of an application $a p$ and $n$ denotes the number of safety applications.

\section{Performance Evaluation}

This paper employs Veins that is a vehicular network simulator [20]. Veins consolidate the network simulator OMNeT++ [21] with the traffic simulator Simulation for Urban Mobility (SUMO) [22]. This simulator can control the flow and interaction in vehicular networks. This paper implements a highway scenario to examine and assess the developed approach. A various number of vehicles has been estimated to depicts the effects of increasing density of vehicles on the performance of tested approaches. The rate of the traffic information has been set up to $10 \mathrm{MHz}$ and the broadcast spectrum has been declared from 300 to 1000 meters. Table I reveals the parameters of the examined simulation scenario. The vehicle's velocity is selected from 20 $32 \mathrm{~m} / \mathrm{s}$ in the motorway scenario.

Table I: Motorway Scenario configuration parameters

\begin{tabular}{cc}
\hline Parameters selection & Values \\
\hline Dimension of motorway road & $850 \mathrm{~m}$ motorway scenario \\
Speed of vehicles & $20-32 \mathrm{~m} / \mathrm{s}$ \\
& \\
Vehicles number & $50,100,150,200$, \\
& 250,300 \\
Simulation time & $250 \mathrm{~s}$ \\
The utilized protocol & WAVE \\
Range of Transmission & $500 \mathrm{~m}$ \\
Rate of transmission & $6 \mathrm{Mbps}$ \\
Information frequency & $10 \mathrm{MHz}$ \\
$\alpha_{\mathrm{j}}$ & 5 \\
$\mu_{\mathrm{j}}$ & 0.7 \\
\hline
\end{tabular}




\section{A. Parameters Selection of NUM-DRC}

Figs 1 and 2 show the impact of varying preferences parameters in the utility function on information rate and COP. Here, when $\mu_{j}$ is fixed to constant values such as 0.7 and $\alpha_{j}$ has various states of $(2.5,5.0$, and 10.0), respectively. Figs 1 and 2 show that the increasing sending rate of $\alpha_{j}$ will enhance the traffic information flow in the network but this comes at the cost of consuming the bandwidth due to the channel capacity limit and broadcasting a large amount of information.

However, improving the preference parameter of $\mu_{j}$ will reduce the damage of competition and will minimize the COP. This is due to apply low information frequency as presented in Figs 3 and 4 . These two figures show the influence of increasing $\mu_{j}$ weight on the COP and information rate when $\alpha_{j}$ has a fixed constant value. Therefore, by adjusting the preference parameter $\mu_{j}$ the information rate and the COP will decline and vice versa. Here, values of the preference parameters $\left(\alpha_{j}\right.$ and $\left.\mu_{j}\right)$ have been determined to obtain a balanced approach that can settle the channel congestion conditions.

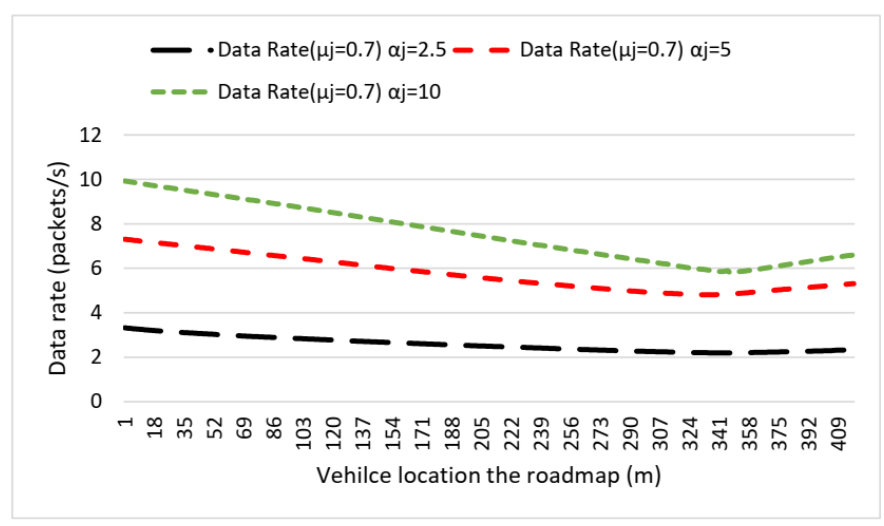

Fig. 1. $\alpha_{j}$ vs information rate

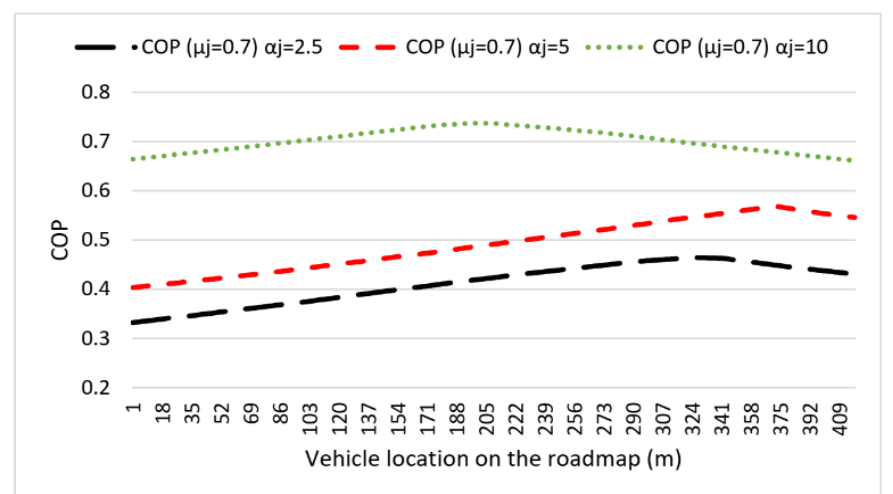

Fig. 2. $\alpha_{j}$ vs COP 


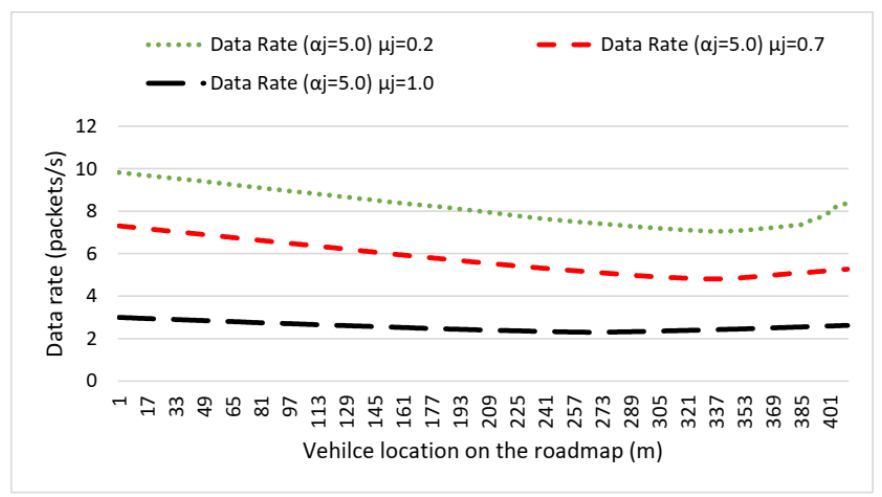

Fig. 3. $\mu_{j}$ vs information rate

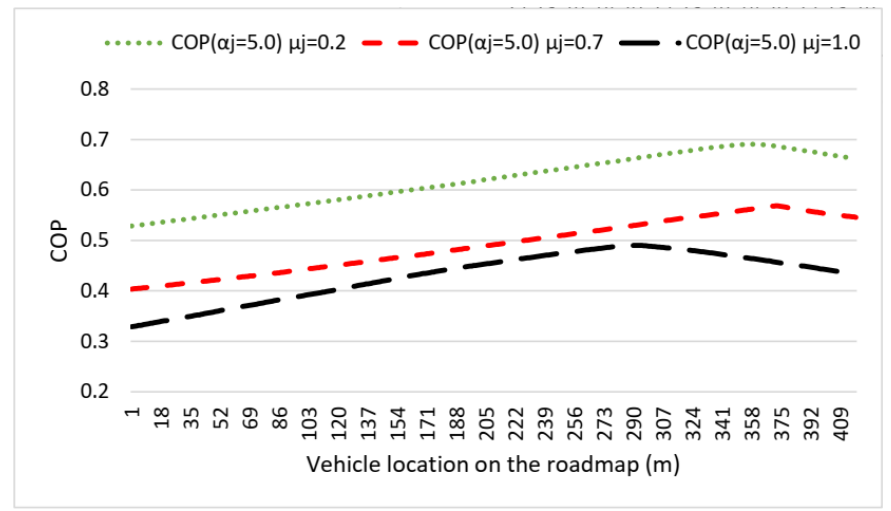

Fig. 4. $\mu_{j}$ vs COP

\section{B. Simulation experiment of NUM-DRC}

The evaluation of performance is employed to determine the effectiveness and ability of the developed broadcast protocol. The common performance properties used by many developed congestion control schemes in VANETs are throughput, packet loss rates, occupancy time, and delay on the channel. A review of measurement parameters is displayed as follows.

1. Throughput: It measures the total amount of collected messages at the end node.

2. Delay: This parameter represents the required time to send a message successfully from the sender application layer to the receiver application layer.

3. Messages Loss: It describes the total amount of the channel and buffer lost messages during the simulation time.

Hither, to measure the performance of the suggested approach, a four-lane motorway road has been created in Veins. Fig 5 reveals the motorway scenario that is performed in Veins. Fig 6 illustrates the impact of increasing of flowing vehicles in the simulation scenario on the throughput. The figure records that there is a concrete connection between the density of communicated vehicles and the amount of successfully received messages at destination vehicles, the throughput rises with an increase of the density of the vehicle's number in the communication environment. It is undeniable that the recorded throughput by the NUM-DRC broadcast protocol is more reliable contrasted to the WAVE and GTACC approaches. This is 
due to the applied efficient adjustment broadcast protocol that aids to alleviate congestion on the wireless channel and reduces the number of dropped traffic information. This supports improving the number of collected messages at the destination vehicles.

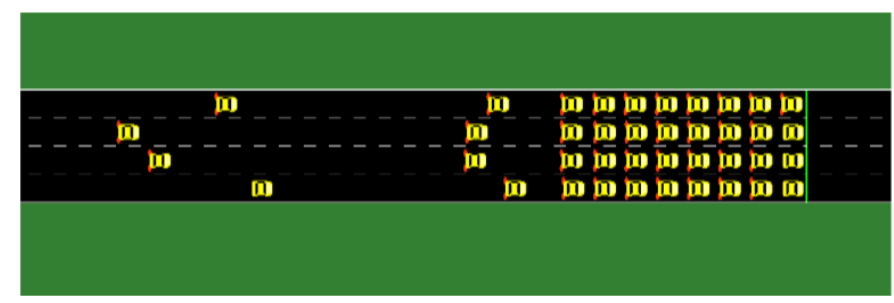

Fig. 5. Motorway scenario for tested approaches

Fig 7 demonstrates the average delay fluctuations effects with increments in the flowing vehicles reported by NUMDRC, WAVE, and GTACC, respectively. According to this figure, it is stated that the increasing density of communicated nodes affects the time delay. Fig 9 points that the time delay values boost when simulation scenario various vehicles number from 50 to 300 . Moreover, this figure has shown that the average delay reported by NUM-DRC is more solid when compared to that one recorded by both the GTACC and WAVE.

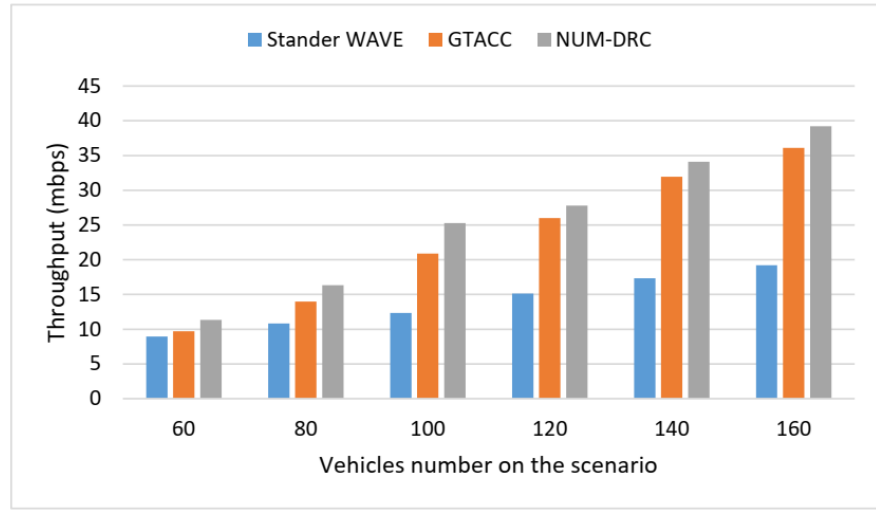

Fig. 6. Throughput of motorway scenario.

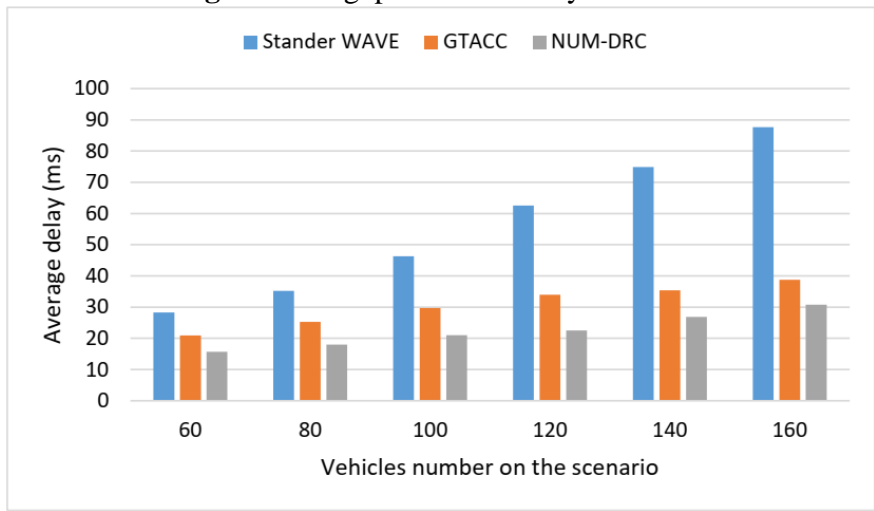

Fig. 7. Average delay of motorway scenario. 
Fig 8 describes the mount of loss packets captured by examined approaches. In the dense environments, the amount of flowing vehicles increases which in turn raises the probability of messages loss. This is because of the increment in the competition among the communicated vehicles to obtain the network channel and transmit information simultaneously which causes to a broadcast storm and crash in data. Figure 10 shows that the NUM-DRC has fewer messages loss in contrasted other approaches due to the fair adjustment broadcast protocol of traffic information rate and considering the $\mathrm{COP}$ as an objective feature which aids to decline the congestion on the channel.

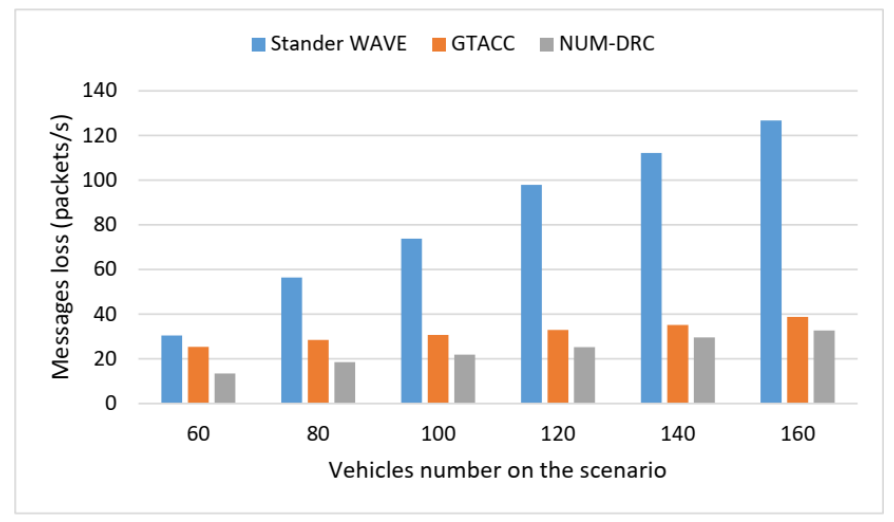

Fig. 8. Messages loss of motorway scenario.

\section{Conclusion}

As the density of communicated vehicles increases on the road segments, the number of disseminated traffic safety messages is also increased which generates channel congestion problem. This diminishes the vehicular network efficiency and quality of service parameters. Therefore, this work develops a novel broadcast rate adaptation strategy that can reduce channel congestion. This approach formulates channel congestion control in vehicular communication as an optimization problem by applying the network utility maximization method to overcome and alleviate the congestion problem. The significance of the developed broadcast protocol on the vehicular network achievement has been recorded by Veins simulation and tested across a motorway scenario as contrasted to the WAVE protocol and GTACC.

\section{Acknowledgments}

The authors are grateful for the support of the Iraqi Government, specialty the Ministry of Higher Education, Southern technical university, and the Technical Institute of Basra.

\section{References}

[1] C. C. C. Pang, W. W. L. Lam, and N. H. C. Yung, "A method for vehicle count in the presence of multiple-vehicle occlusions in traffic images," IEEE Transactions on Intelligent Transportation Systems, vol. 8, no. 3, pp. 441-459, 2007.

[2] S. Sharma and S. Sebastian, "IoT based car accident detection and notification algorithm for general 
road accidents.” International Journal of Electrical \& Computer Engineering (2088-8708), vol. 9, 2019

[3] W. Viriyasitavat, T. Anuphaptrirong, and D. Hoonsopon, "When blockchain meets internet of things: characteristics, challenges, and business opportunities," Journal of industrial information integration, 2019.

[4] C. R. Storck and F. Duarte-Figueiredo, "A $5 \mathrm{~g}$ v2x ecosystem providing internet of vehicles," Sensors, vol. 19, no. 3, p. 550, 2019.

[5] Z. Tong, H. Lu, M. Haenggi, and C. Poellabauer, "A stochastic geometry approach to the modeling of dsrc for vehicular safety communication," IEEE Transactions on Intelligent Transportation Systems, vol. 17, no. 5, pp. 1448-1458, 2016.

[6] J. Singh and K. Singh, "Congestion control in vehicular ad hoc network: A review," in NextGeneration Networks. Springer, 2018, pp. 489-496.

[7] L. Wischhof, A. Ebner, H. Rohling, M. Lott, and R. Halfmann, "Adaptive broadcast for travel and traffic information distribution based on intervehicle communication," in Proceedings of the IEEE IV2003 Intelligent Vehicles Symposium, 2003. IEEE, 2003, pp. 6-11.

[8] R. K. Schmidt, T. Leinmuller, E. Schoch, F. Kargl, and G. Schafer, "Exploration of adaptive beaconing for efficient intervehicle safety communication," IEEE network, vol. 24, no. 1, 2010.

[9] M. I. Hassan, H. L. Vu, and T. Sakurai, "Performance analysis of the ieee 802.11 mac protocol for dsrc safety applications," IEEE Transactions on vehicular technology, vol. 60, no. 8, pp. 3882 3896, 2011.

[10] D. Puthal, Z. H. Mir, F. Filali, and H. Menouar, "Cross-layer architecture for congestion control in vehicular ad-hoc networks," in Proceedings of International Conference on Connected Vehicles and Expo (ICCVE), 2013. IEEE, 2013, pp. 887-892.

[11] H. M. Amer, C. Tsotskas, M. Hawes, P. Franco, and L. Mihaylova, "A game theory approach for congestion control in vehicular ad hoc networks," in Proceedings of IEEE Sensor Data Fusion: Trends, Solutions, Applications (SDF), 2017, pp. 1-6.

[12] F. Gebali, Analysis of computer networks. Springer, 2015, NewYork, USA.

[13] S. Keshav, Mathematical foundations of computer networking. Addison-Wesley, 2012, Iniana, USA.

[14] Q.-T. Nguyen-Vuong, Y. Ghamri-Doudane, and N. Agoulmine, "On utility models for access network selection in wireless heterogeneous networks," in NOMS 2008-2008 IEEE Network Operations and Management Symposium. IEEE, 2008, pp. 144-151.

[15] J. K. MacKie-Mason and H. R. Varian, "Pricing congestible network resources," IEEE journal on Selected Areas in Communications, vol. 13, no. 7, pp. 1141-1149, 1995.

[16] Q. Chen, D. Jiang, T. Tielert, and L. Delgrossi, "Mathematical modeling of channel load in vehicle safety communications," in 2011 IEEE Vehicular Technology Conference (VTC Fall). IEEE, 2011, pp. 15.

[17] D. P. Bertsekas and A. Scientific, Convex optimization algorithms. Athena Scientific Belmont, 2015.

[18] D. P. Palomar and M. Chiang, "Alternative distributed algorithms for network utility maximization: Framework and applications," IEEE Transactions on Automatic Control, vol. 52, no. 12, pp. 2254 2269, 2007.

[19] Q.-V. Pham and W.-J. Hwang, "Network utility maximization-based congestion control over wireless networks: A survey and potential directives," IEEE Communications Surveys \& Tutorials, vol. 19, no. 2, pp. 1173-1200, 2016.

[20] Muniyandi RC, Qamar F, Jasim AN. Genetic Optimized Location Aided Routing Protocol for VANET Based on Rectangular Estimation of Position. Applied Sciences. 2020 Jan; 10(17):5759.

[21] Varga A. A practical introduction to the OMNeT++ simulation framework. InRecent Advances in Network Simulation 2019 (pp. 3-51). Springer, Cham.

[22] Behrisch M, Bieker L, Erdmann J, Krajzewicz D. SUMO-simulation of urban mobility: an overview. In Proceedings of SIMUL 2011, The Third International Conference on Advances in System Simulation 2011. 